\title{
Investigating how university students collaborate to compose physics problems through structured tasks
}

\author{
Javier Pulgar $\odot^{*}$ \\ Departamento de Física, Universidad del Bío Bío, 4051381 Concepción, Chile \\ Valentina Fahler® and Alexis Spina® \\ Gevirtz Graduate School of Education, University of California Santa Barbara, \\ Santa Barbara, 93106-9490 California, USA
}

(Received 7 January 2020; accepted 25 January 2021; published 29 March 2021)

\begin{abstract}
Traditionally, scholars in physics education research have focused on students solving well-structured learning activities at the university. However, due to their constrained nature, these problems hinder collaboration and idea generation. In order to encourage student collaboration and decision making demands among undergraduate students in an introductory physics course, we utilized a real-world problem where groups were asked to compose a well-structured physics problem for younger learners. In this study we explored how they collaborated in composing physics problems. Data collection consisted of audio recording of the group discussions while they were collaborating to develop their physics problems and their respective problem solutions. Through interviews, we accessed participants' perceptions of the task and related challenges. Results suggest that composing problems is an opportunity for participants to propose ideas and make decisions regarding the goals of the problem, concepts and procedures, contextual details, and magnitudes and units to introduce in their activities. Further, participants valued the openended nature of the task and recognized its benefits in utilizing physics ideas into context, which in turn enabled collaboration in a way not experienced by them with traditional well-structured problems. These findings shed light on the nature of composing physics problems in undergraduate courses, where participants assume a different role by facing a real-world activity that encourages expertise through physics-related communication and writing.
\end{abstract}

DOI: 10.1103/PhysRevPhysEducRes.17.010120

\section{INTRODUCTION}

Physics education has long relied on problem solving as a mechanism to foster student learning. In general, the problems used have been classified within two broad categories, namely, well-structured and real-world problems. While prevalent in physics curriculum, physics education research (PER) scholars have lately found that well-structured physics problems have little effect in students' conceptual development $[1,2]$ and collaboration opportunities $[3,4]$. The consistent engagement with wellstructured physics problems fosters superficial problemsolving strategies (e.g., "plug and chug"), which discourage students from reflecting upon the physics concepts and

*Departamento de Física, Universidad del Bío Bío, Avda Collao 1202, Concepción, Chile.

jpulgar@ubiobio.cl

Published by the American Physical Society under the terms of the Creative Commons Attribution 4.0 International license. Further distribution of this work must maintain attribution to the author(s) and the published article's title, journal citation, and DOI. principles, and thus limits their conceptual growth [1,2]. Students solving well-structured problems experience low levels of positive interdependence (i.e., collective perception that group performance enhances benefits and success) $[5,6]$, which motivates the individual rather than collaborative performance $[7,8]$. On the contrary, PER scholars have found that using real-world problems in the classroom can potentially create opportunities for students to engage in problem-solving skills, and increase conceptual development and decision making $[9,10]$. The learning opportunities that real-world problems promote have a great importance for the PER field, which must rapidly adjust to the fast and ever-changing professional responsibilities that their students will encounter after graduation.

Real-world problems present learning situations that emerge from real-life experiences and/or demands, such as composing problems for younger learners. These problems are characterized by having low numbers of problem-related constraints, and therefore a multiplicity of pathways towards solutions [6]. The PER literature on the subject has found that success with real-world problems is achieved when students display an appropriate 
knowledge domain, the ability to regulate thoughts and beliefs, and to justify ideas and/or decisions [11]. These findings have been extended by studies about context-rich problems, which support the importance of having experience in dealing with unfamiliar situations [12], and effective group processes [4].

While existing studies have clearly established the value of introducing real-world problems into the physics classroom, there is still little information about the dynamics of collaboration among students when composing (i.e., writing) physics problems, specifically when it comes to the negotiation of physics concepts and task related ideas. This paper describes the benefits and challenges experienced by undergraduate physics students when composing realworld problems in groups. We explored the physics related ideas and various collaborative processes involved in a physics writing-to-learn (WTL) activity. The results of this study suggest that composing real-world problems in the physics classroom promotes discussions about the goals of the problem, concepts and procedures, contextual details, and magnitudes and units to introduce in their activities. In addition, evidence shows that participants decided on the concepts and procedures through a combination of mechanisms defined as follows: equation-driven approach and concept-driven approach, two mechanisms that mimic problem-solving strategies associated with both novices and experts. Finally, even though participants admitted to having little experience with solving real-world problems, they valued the open-ended nature of the task and highlighted the positive learning outcomes of group discussions that led to the application of physics ideas in a specific context.

This paper is structured as follows: First, we cover the literature on problem solving in physics and collaboration. Next, we view problem solving through the lens of WTL notions about composing group problems and how and why these are important questions to consider when presenting students with a disciplinary-based writing task. Then, we report on the data and connect the findings to current discussions about student learning in physics, conceptual development, and collaboration. Our findings suggest that using real-world problems that are framed within tasks that clearly state real audiences and contexts, expectations, procedures, and require group discussions can positively support student learning in the discipline.

\section{COMPOSING PHYSICS PROBLEMS AND EXPERTISE}

The relationship between conceptual development and disciplinary enculturation at the undergraduate level has been an ongoing discussion among education scholars. From a situated learning perspective [13], learning has been framed as a process that happens through participation in a community, and its importance extends far beyond the acquisition of skills and knowledge. When engaging in the specialized activities of a community, the individual gains a social position in the community as well as an identity $[14,15]$. Longitudinal studies of undergraduates engaging in the work of different disciplines have shown this identity shift from being novices to more experienced members of their disciplines, examples being in the social sciences [16], science, technology, engineering, and mathematics (STEM) disciplines [17,18] and in humanities [17]. In all of these cases, students' transformation was accompanied by a more nuanced understanding of disciplinary core ideas; in Meyer and Land's terms [19], the disciplines' threshold concepts, namely, those concepts that once acquired, changed completely how the discipline was understood. Naturally, every discipline has specific ways of familiarizing students with their threshold concepts.

In PER, scholars have been researching the learning consequences of students engaging in different disciplinary-based tasks. Overall, studies found that solving traditional well-structured problems does not necessarily lead to adequate conceptual development [1,2]. According to Byun and Lee [1,2], the best predictors of conceptual development are the strategies used for solving the problemsrather than the number of problems solved. Expert problem solvers usually tackle problems drawing on knowledge development; that is, they first build an extensive conceptual understanding before they attempt to solve a problem [2]. Alternatively, less experienced problem solvers follow a means-end strategy; they focus first on the problem's goals before attempting to build conceptual meanings, a strategy that makes them work backwards and overlook the meanings associated to solving the activity [3,20]. In general, well-structured problems tend to encourage means-end rather than knowledgedevelopment strategies, because students frequently focus on finding the equation that best fits the problem, a behavior typically associated with novice solvers $[1,2,21]$. This equation-driven approach enacted by novices has also been labeled as bottom-up logic [22,23], whereas experts are more likely to engage with top-down strategies: they begin from general principles and then move down to mathematical representations and equations that enable them access to the solution [20,21].

In the same line, qualitative physics problems can provide a rich opportunity for students' learning [23-25] as well as collaboration [26,27]. More specifically, PER scholars have explored the consequences of students composing qualitative physics problems and have found positive learning outcomes. In traditional undergraduate physics courses this practice is usually unfamiliar to the students, who have been trained since high school to solve well-structured problems. For instance, Hardy and colleagues [28] designed a course where students periodically were asked to create their own multiple-choice questions. This activity resulted in positive learning outcomes, especially for low and middle performance course participants. 
According to Mestre [29], composing questions is a cognitively demanding task that sheds light on the development of students' expertise and knowledge transfer into different contexts. It can also help diagnose the stage of conceptual development of the learners, a useful pedagogical tool that can guide further instructional decisions. In this case, students' productions might reflect failings and limitations of given instructional strategies-for example, if students' problem-generation process relies mostly on procedural than on declarative knowledge [29].

It is also important to point out that the writing-to-learn (WTL) literature has found that the very act of writing within disciplinary settings fosters intellectual development $[30,31]$. When students write physics problems, they face the challenge of making appropriate choices in relation to the genre (i.e., context, audience, writer) [32] and in its content (variables, questions, formulas) [33]. In addition, writing is a creative process where writers draw on linguistic and cultural resources to develop new thoughts [34,35]. Specifically in science education, the most beneficial writing assignments have been found to be those that state explicit goals; that prompt critical thinking, creativity and application of knowledge; assignments that foster collaboration among peers; and that encourage decision making [18]. Overall, these types of writing activities engage students in knowledge transforming processes [36]. Recent examples from science instructors operating within this WTL framework include drawing assignments in the biology classroom [37], composing memes in a psychology course [38], or writing science articles for nonacademic audiences in biology and molecular biology courses [39].

In physics, composing problems is a real-world task $[12,29]$ frequently engaged by teachers and instructors. A real-world problem requires solvers to have the ability to generate subjective assumptions over issues relevant to the problem context, in order to transform and constrain the open-ended scenario into a well-structured one $[12,40]$. These real-world problems are designed for the highest level of cognitive demand, known as knowledge utilization [9,41], which include competencies associated with idea generation and decision making, a set of processes that benefit from collaboration $[42,43]$. Having been exposed to such cognitive demand is what separates novices and experts when successfully solving real-world problems in physics [12]. According to Fortus [12], when solving real-world physics problems, the hardest choices to make are associated with the absolute or relative magnitudes of the variables involved, a practice rarely found in introductory courses and related tasks. In contrast, easier choices relate to physics variables and principles involved in the problems [12]. In his study Fortus did not consider, however, the rhetorical demands that the task imposes to the students.

When novices are assigned a task that is usually carried out by more expert members of the community, they are encouraged to think like experts would. In the case of students writing physics problems, they are asked to imitate the thinking processes and discursive choices of their instructors, a form of mimicry that could lead to a more sophisticated understanding of the subject matter, creativerelevant skills, and prompt new disciplinary questions [19]. Furthermore, creative problem solving also requires knowledge-relevant skills [44], or in other words, an appropriate understanding of the subject matter and related procedures involved in creative thinking. In our study the content relates to circular motion, a phenomena that has been found to be challenging for teachers and students, particularly due to misconceptions around the idea of inertia [45-47].

This section presents theories and empirically based research that indicated the benefits of composing physics problems for students' disciplinary enculturation. Specifically, the open-ended and real-world nature of the task, as well as its written component seem to provide rich learning opportunities, and promote creativity and problem solving. Since asking students to engage in expertlike behaviors is a challenging endeavor, student collaboration becomes critical. The following section covers in more detail the theoretical underpinnings of collaboration and problem solving, and also reviews relevant studies that have addressed this issue in physics education.

\section{A. Collaboration and problem solving}

Although much of the learning that happens within a classroom is centered around the individual's performance (e.g., in exams, readings, lectures), from a sociocultural perspective, it is through collaboration with peers that learning and development happens. Therefore, this framework explores learning not as an individual endeavor, but instead as a collaborative process with peers, which activates what is known as the zone of proximal development (ZPD) [48]. Vygostky points out that when an individual collaborates with peers in a challenging task, they learn new ways of solving problems that subsequently lead to a cognitive reorganization and transformation (i.e., development) in the subject matter [34].

This way of thinking about learning has led to many studies that compared an individual performance in a task versus the group performance. In mechanics, students facing context-rich problems in groups provided better problem solutions than individuals working alone [3,4]. Furthermore, students' discussion for problem solving has prompted engagement with problem-solving strategies typically associated with experts, such as qualitative descriptions of the phenomena $[26,27]$ and learning gains [49]. Equally important, undergraduate students across disciplines have reported that projects that enable collaboration with peers constitute more meaningful learning experiences [50].

In the context of problem solving as a group activity, research has found that collaboration operates differently 
depending on the nature of the task. For instance, wellstructured physics problems might be associated with disjunctive activities [7], as these do not necessarily demand collective efforts to find the right solution. Contrarily, the activity of composing physics problems might be associated with additive tasks [7], because a good performance would likely emerge from the sum of all members' contributions and relevant abilities. This creative problem would present higher levels of positive interdependence [5] in contrast to algebra-based tasks, as its solution stemmed from collective contributions [51-55].

The collective process of coming up with ideas to compose physics problems remains an unknown territory for educators, as we still do not know what type of ideas students engage in for decision making and their strategies and mechanisms for collaboration. Therefore, in this study we explore how groups work together to compose a physics problem for an audience of high school students. In doing so, we attempt to answer the following research questions:

RQ1: What are the physics and task related ideas that participant groups addressed when composing a physics problem for high school students?

RQ2: What are the perceived benefits and challenges of composing a physics problem for high school students?

RQ3: How do participant groups collaborate when composing a physics problem for high school students?

\section{METHODS}

\section{A. Research contexts and participants}

This qualitative study was conducted in two sections from an undergraduate introductory physics course at a private university in Chile. The course curriculum addressed contents such as vector algebra, kinematics, Newton's laws, and rotational dynamics. Each course section was taught by a different physics professor, who covered the same curriculum during two weekly lecturebased sessions, and one problem-solving session, where participants typically worked in groups to solve wellstructured and real-world physics problems. Section I had 34 participants (8 females, 26 males), and Sec. II had 37 participants (13 females, 24 males). All of the research participants pursued a college degree in engineering and were in their first or second undergraduate year. The course was taught in Spanish, the participants' native language.

As part of a larger research project, instructors were assigned two alternative strategies to guide the problemsolving sessions. The instructor froproblem-solving Sec. I was asked to guide the problem-solving session by highlighting the importance of creativity and social interactions, and responded to participants' questions by guiding them towards classmates who could have the answer. The instructor in Sec. II made no reference to creativity for composing the physics problem and responded to participants' questions by providing direct information.

During a problem-solving session on the seventh week of the semester, we presented participants with the activity in Fig. 1. As shown in Fig. 1, the prompt first presented the rhetorical strategies (e.g., describe, plan, define) that participants should follow. These strategies served to scaffold the students' composing process, considering their minimal experience performing as writers of physics problems. Additionally, the activity was contextualized within a concrete scenario and a specific audience (i.e., a high school classroom, their teachers and students), all important elements from a WTL perspective [56]. At the same time, students were asked to both apply conceptual knowledge and plan, monitor and evaluate the choices they made. Lastly, following the recommendations from Heller and Hollabaugh [3], we formed groups of 3 to 4 participants, as this group size has shown to perform better than pairs and larger groups in solving context-rich problems.

Overall, the characteristics of the activity aligned with what Gere and colleagues [18] found as key dimensions of writing assignments that promote effective learning in science; namely, meaning making, interactions, clear expectations, and metacognition. This was a meaningmaking activity because it asked participants to integrate their physics knowledge and adapt it creatively in the form

\section{Task for Data Collection}

To solve the following problem assigned to your group, consider the following strategies:

- Describe the problem in terms of physics concepts and principles.

- Plan and define a strategy to develop a solution.

- Build on your strategies to execute the solution.

- Check and assess your results and appropriateness of your solution.

Problem:

As part the university community, you and your team have been selected to teach the kinematics of circular motion to high school students. It is known that individuals are capable of understanding new content and information when it is contextualized or applied and connected to daily situations. Physics and math teachers from the school where your team will teach the physics of circular motion have been preparing their students in the needed mathematical content to ease their physics learning. With this, your team is responsible for identifying daily situations that would enable the study of circular motion and create a math-based problems for high-school students to solve.

FIG. 1. Real-world physics problem administered to course participants. 


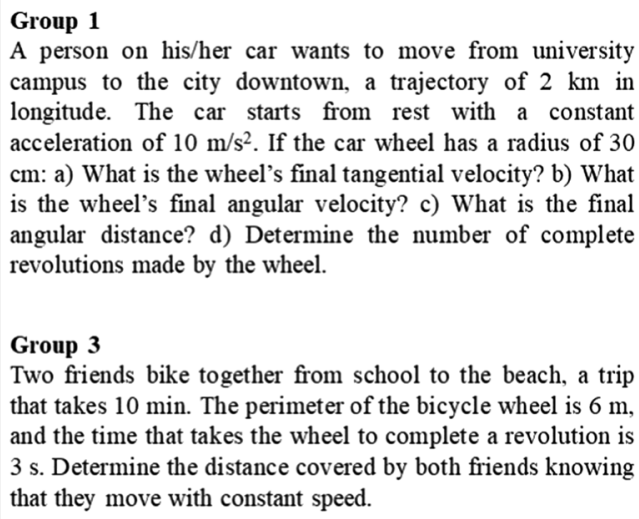

Group 2

A group of college students had the brilliant idea to put one of their peers into a $1 \mathrm{~m}$ radius barrel and push them in it. With such enthusiasm, students could make the barrel spin with an angular acceleration of $2 \mathrm{rad} / \mathrm{s}^{2}$. If the initial angular speed was $2 \mathrm{rad} / \mathrm{s}$, respond: a) If the student inside the barrel reaches more than 150 complete revolution in the first $30 \mathrm{~s}$, he/she will vomit. Is it possible for them to reach this?; b) Determine the angular and tangential speed at $30 \mathrm{~s}$.

Group 4

Donkey Kong wants to throw barrels to King K Rool. For this, Donkey Kong throws one with an angular speed of $2 \pi$ $\mathrm{rad} / \mathrm{s}$. By knowing that at $3 \mathrm{~s}$ its speed is $10 \pi \mathrm{rad} / \mathrm{s}$, and that the barrel impacts at $5 \mathrm{~s}$, determine: a. the angle covered by the barrel; b. the magnitude of the centripetal and tangential acceleration at the moment of impact at $6 \mathrm{~cm}$ from its center; c. frequency and period.

FIG. 2. Problems generated by groups 1, 2, 3, and 4 .

of a problem for high school students. At the same time, participants needed to compose these problems in groups, which promoted collaboration. When participants needed to negotiate choices within their own groups, they had to verbalize and explain their thoughts to the group to reach consensus, an action that fosters metacognition-defined as conscious awareness of mental processes [57]. The activity listed clear expectations by providing information about the genre (a physics problem), the audience and context (high school students), the concepts (kinematics of circular motion), and the steps to follow. In addition, the activity asked participants to rely on their own experiences_or "funds of knowledge" [58] — to complete the activity, since the participants themselves had previously been high school students who would solve physics problems.

Our study focuses on four of the groups, two from each section. In Sec. I, groups G1 (2 female and 2 male participants) and G2 (4 male participants), and from Sec. II, groups G3 (2 female and 1 male participants) and G4 (4 male participants). The group selection was based on the instructors' experience working with the students. According to the instructors, these were the 4 groups that had displayed the best performance solving previous tasks. We had no access to additional participants' information in terms of their age, race, socioeconomic background, or grades. Figure 2 depicts the physics activities composed by these four groups. All of the problems fall under the category of well structured, and with various levels of originality and appropriateness for younger students. A more detailed analysis of the physics problems composed by these groups and the rest of the class participants can be found in Ref. [33].

\section{B. Data collection and analysis}

The present study is informed by two data sources: audio recordings from the group problem-solving session and individual interviews with group members. The former provided information about the disciplinary content, problems, and negotiations among participants as the activity developed; while the latter added more specific information about the characteristics, strategies, and challenges that the participants experienced when solving the activity. Both data sources were collected to provide evidence for RQ1 (audio recordings), and RQ2 and RQ3 (individual interviews).

First, we audio recorded $1.5 \mathrm{~h}$ of the interactions from groups G1, G2, G3, and G4 as they were solving the activity. A total of 295 min of audio recording were transcribed by identifying speech turns. We understand speech turns from a conversational analysis perspective, namely, utterances that are not only responses to previous utterances but also prompt future responses [59]. For example, Participant 1: How did you obtain the number of revolutions? Did you multiply the number by something?; Participant 2: There is one revolution and two revolutions. This example illustrates two speech turns: the first turn is comprised by the questions from participant 1 ; after participant 1 voiced his questions (a sign of the end of his speech turn), participant 2 takes on the speech turn and answers the questions. The day after the session, we asked one group member per team to volunteer for a 15-20 min interview. In total, we carried out 4 interviews. The interviews were semistructured, a choice that is flexible enough to understand how each participant makes meaning of the experience in their own terms and, at the same time, provides a structure to compare the information among the different participants [60]. In the interview, participants were asked about their experience composing physics problems, benefits, challenges and the way they collaborate with their teammates and others (see interview protocol in the Supplemental Material [61]). The interview protocol was also designed to elicit the differences between wellstructured and real-world problems. The analysis of group recordings and interviews was conducted in Spanish, and the quotes presented as evidence were translated to English. 
TABLE I. Code book of emergent categories and themes addressed by 4 participant groups during the task of composing a physics problem.

\begin{tabular}{|c|c|c|}
\hline Code & Description & Example \\
\hline \multicolumn{3}{|c|}{ Problem definition } \\
\hline Audience and learning goals & $\begin{array}{l}\text { Team discusses and makes decisions regarding } \\
\text { the learning goals for the generated problem, } \\
\text { and the expectation of what the targeted } \\
\text { students should learn from it, which mediates } \\
\text { the degree of difficulty taking in } \\
\text { consideration the school level of the targeted } \\
\text { students. }\end{array}$ & $\begin{array}{l}\text { G1: What is the goal of this (activity)? I mean, of } \\
\text { teaching this?; It would be like explaining them } \\
\text { (high school students) how these (movements) } \\
\text { work. }\end{array}$ \\
\hline $\begin{array}{l}\text { Physics concepts and } \\
\text { procedures }\end{array}$ & $\begin{array}{l}\text { Team identifies, poses and decides on the } \\
\text { physics concepts to use into the problem, as } \\
\text { well as the ways in which these concepts } \\
\text { align with the generated problem to be well } \\
\text { structured, and consistent with the task } \\
\text { requirements. }\end{array}$ & $\begin{array}{l}\text { G1: I supposed we need to include the equations. } \\
\text { That way they only need to replace; So the } \\
\text { problem must be in order. First you calculate } \\
\text { one (value), which then allows you to find } \\
\text { another. }\end{array}$ \\
\hline $\begin{array}{l}\text { Problem context and } \\
\text { wording }\end{array}$ & $\begin{array}{l}\text { Team poses and decides on the } \\
\text { contextualization of the problem (i.e., place, } \\
\text { subjects, actions, etc.), and the wording of the } \\
\text { problem. }\end{array}$ & $\begin{array}{l}\mathrm{G} 4: \text { Let's do something cool, like a wooden } \\
\text { spinning top.; G1: If I want to say that the car } \\
\text { wants to move from A to B, is that displacement? }\end{array}$ \\
\hline $\begin{array}{l}\text { Discussing magnitudes } \\
\text { and units }\end{array}$ & $\begin{array}{l}\text { Team discusses and decides the magnitudes } \\
\text { scores and values, as well as measurement } \\
\text { units for the physics concepts (e.g., } 10 \mathrm{~km} / \mathrm{h} \text {, } \\
20 \mathrm{~s}, 2.5 \mathrm{~km} \text { ) to be introduced into the } \\
\text { problem's description. }\end{array}$ & $\begin{array}{l}\text { G2: How much do we say the acceleration will be?; } \\
\text { G1: Do you want the car to get to its destination } \\
\text { fast or slow? }\end{array}$ \\
\hline Algebraic procedures & $\begin{array}{l}\text { Problem solving strategies } \\
\text { Team describes algebraic steps to obtain } \\
\text { physical quantities as a way of solving the } \\
\text { problem, normally mentioned to justify the } \\
\text { appropriateness of the designed problem. }\end{array}$ & $\begin{array}{l}\text { G4: Because you have the angular speed at } 3 \mathrm{~s} \text {, } \\
\text { which is } 10 \pi \text {, so } 10 \pi \text { is equal to (angular) } \\
\text { acceleration plus the initial angular speed. } \\
\text { So then you clear and get the (angular) } \\
\text { acceleration. }\end{array}$ \\
\hline $\begin{array}{l}\text { Physics of circular motion } \\
\text { in context }\end{array}$ & $\begin{array}{l}\text { Team engages in a qualitative description of the } \\
\text { physics regarding the circular motion in the } \\
\text { context under consideration for the problem. }\end{array}$ & $\begin{array}{l}\text { G3: There is also velocity, this velocity that goes to } \\
\text { the middle. This is the one that enables... This } \\
\text { was related to forces if I remember. The topic of } \\
\text { the two forces pointing out to one side. }\end{array}$ \\
\hline
\end{tabular}

This translation was checked by the research team, which includes two bilingual speakers who were Spanish native speakers, and one English native speaker.

Because of the linguistic nature of the data, we chose to carry out the analysis of both the group sessions and the interviews applying qualitative coding techniques. From a qualitative research standpoint, the coding of verbal data can be defined as "the analytic task of placing non-numeric data into descriptive categories, assigning them to codes" [62] (p. 3). We segmented the transcribed data into topical chains, defined as units of discourse consisting of clauses organized around a topic [62]. This choice enabled us to capture the different ideas that the participants were bringing into their understanding of physics problems. To identify the borders among the ideas, we relied on linguistic resources. For example, as an answer to the interview question about the process of solving well-structured problems, participant 1 answered: "[When I face a well-structured problem, my goal is to solve it. At that moment, I barely reflected. Since I have the data, I use that data in the given formulas and that's it. I obtained my result.] [However, when facing an real-world problem....]." In this excerpt, we identified two topic chains. The first one structured around the cognitive processes of solving wellstructured problems and the second one, around the realworld ones. In the previous example, it is the transitional device "however" that clearly established the beginning of a new topic chain.

After segmenting all the data, coding was conducted in stages assisted by NVivo 12 plus software. First, we coded the data from the group activity drawing on grounded theory [63] techniques. A grounded theory approach stresses the situated and contingent meanings present in the data. Thus, it enabled us to adopt an emic perspective $[60,64]$. An emic perspective underscores the conceptual schemes, words and categories that participants rely on, and 
TABLE II. Code book for analysis of participants' interviews.

\begin{tabular}{|c|c|c|}
\hline Code & Description & Examples \\
\hline $\begin{array}{l}\text { Features of well-structured } \\
\text { problems }\end{array}$ & $\begin{array}{l}\text { Makes reference to attributes, advantages, } \\
\text { challenges and strategies for solving } \\
\text { well-structured physics problems }\end{array}$ & $\begin{array}{l}\text { Part.K: ...are daily life situations, but with concrete } \\
\text { information so there nothing to do, so you go } \\
\text { and replace and use the equation and it is ready; } \\
\text { Part.G: It is mechanic, like you do not make the } \\
\text { effort to understand it, bur rather try to get the } \\
\text { pattern }\end{array}$ \\
\hline $\begin{array}{l}\text { Features of real-world } \\
\text { problems }\end{array}$ & $\begin{array}{l}\text { Makes reference to attributes, advantages, } \\
\text { challenges and strategies for solving } \\
\text { real-world physics problems }\end{array}$ & $\begin{array}{l}\text { Part.B: It is harder because we are not use to create } \\
\text { problems on our own; Part.J: I believe that the } \\
\text { real-world problem is better, because you have } \\
\text { to think harder and realize stuff }\end{array}$ \\
\hline $\begin{array}{l}\text { Collaboration for solving } \\
\text { well-structured problems }\end{array}$ & $\begin{array}{l}\text { Makes reference to the collaboration strategies } \\
\text { for solving well-structured physics problems }\end{array}$ & $\begin{array}{l}\text { Part.K: If we are on the same group we try to solve } \\
\text { it individually. Then if one gets stuck then would } \\
\text { ask other member, and this member gets stuck } \\
\text { would ask, and like that }\end{array}$ \\
\hline $\begin{array}{l}\text { Collaboration for solving } \\
\text { real-world problems }\end{array}$ & $\begin{array}{l}\text { Makes reference to the collaboration strategies } \\
\text { for solving real-world physics problems }\end{array}$ & $\begin{array}{l}\text { Part.B: We all have to pay attention or we may miss } \\
\text { something; Part.J: Here we did all together. For } \\
\text { this problem (composing a problem) at least, we } \\
\text { all gave ideas }\end{array}$ \\
\hline
\end{tabular}

strives to build a description of the phenomena based on the participants' experiences. At the same time, an emic approach opposes an etic approach, because the latter revolves around the researchers' categories and experiences.

Initially, we reviewed $25 \%$ of the group discussion data, using segment by segment coding [63], and identified emergent issues and ideas participants discussed for solving the problem by attending to the dimensions that require decision making, and strategies for solving physics problems (i.e., algebra, conceptual understanding of circular motion). This exploratory analysis led to a first coding scheme with 12 emergent themes associated with different problem-solving stages and ideas for composing a problem.

When we revised this initial coding scheme, we noticed that some codes emerged as part of broader issues within the group's discussion, and thus decided to merge similar codes. For example, after the first analysis we identified that participants regulated the complexity of their problem-regulating complexity, by positioning themselves in what the targeted students would do for solving their problem-behavioral expectations, and then trying to achieve the learning goals of the activity-learning goals. Consequently, we considered the learning goals in relation to the audience to be the broader theme that linked complexity and expectations, and we decided to merge them into one code labeled as "audience and learning goals." We followed this rationale with closely linked codes and came up with the final code book shown in Table I.

After revising the code book, we met with a doctoral researcher trained in qualitative coding, discourse, and text analysis whose first language is Spanish to review and redefine the code book. We first explained the code book and showed prototypical examples from the $25 \%$ of data initially analyzed. We then coded together $15 \min (6.25 \%)$ of the transcribed group discussion data and discussed any disagreement. Finally, the doctoral researcher and the principal investigator coded independently $45 \mathrm{~min}$ $(18.25 \%)$ of the data, obtaining a Cohen's kappa of 0.94 for interrater reliability.

The emergent themes from the group discussion data were used as the basis for the analysis of the participants' interviews. More specifically, from the group discussion themes we created a coding scheme (see Table II) that allowed us to identify the following in the interviews: (i) The perceived benefits and challenges of composing a problem; and (ii) the nature of the collaboration among participants for composing a physics problem. Once the four interviews were coded, we met again with the same doctoral researcher to measure interrater reliability. Similar to what was done before, we discussed the coding scheme, coded together one interview, and then the doctoral researcher coded independently the remaining 3 interviews. The Cohen's kappa obtained was 0.93 for interrater reliability.

\section{RESULTS}

\section{A. Physics and task related ideas for composing a problem}

In the analysis of group recordings we identified two major categories that emerged during the activity: (a) problem definition and (b) problem-solving strategies. As described below, the problem definition category includes processes related to decision making and idea generation that groups enacted in the activity. Here, we observed 
TABLE III. Categories and themes addressed by 4 participants groups during the task of generating a physics problem for high school students, and number of times (percentages) coded in the aggregated data.

\begin{tabular}{lc}
\hline \hline Categories and themes & Frequency (\%) \\
\hline Problem definition & \\
Audience and learning goals & $25(4.2 \%)$ \\
Physics concepts and procedures & $209(34.9 \%)$ \\
Problem context and wording & $128(21.4 \%)$ \\
Discussing magnitudes and units & $90(15.1 \%)$ \\
Problem solving strategies & \\
Algebraic procedures & $98(16.4 \%)$ \\
Physics of circular motion in context & $48(8.0 \%)$ \\
Total & $598(100 \%)$ \\
\hline \hline
\end{tabular}

questions about the audience and learning goals; the physics concepts and procedures; the problem context and the wording; and the magnitudes and units used. The other category, problem-solving strategies, includes processes related to physics and mathematics-specific concepts and representations, and typically related to solving well-structured problems. Within this category, two main themes were discussed: algebraic procedures and steps; and physics of circular motion. Table I lists, describes, and provides examples of each of these themes. The following subsections expand on these ideas and illustrate them with excerpts from the data.

\section{Problem definition}

The first category (problem definition) refers to processes related to making decisions and generating ideas in order to create the physics problem. During these processes, teams discussed and decided on issues, such as the learning goals of the activity, the concepts and procedures, and the contextual details of how these elements would be presented in written form. They also decided on the necessary data to create a well-structured problem and the appropriate questions to ask in relation to their data. In Table III, readers might notice that groups spent most of their time addressing themes related to problem definition, which included approximately $75 \%$ of the message units analyzed.

It is important to note a similarity between the themes that emerged in this category and the set of ideas instructors frequently decide on when they create pedagogical material. For example, the audience and learning goals, content and processes embedded in the activity to encourage thinking and knowledge building, and the pieces of information that make the task appealing (i.e., context) and well-structured (i.e., data). In the following paragraphs we describe the nature and details of the ideas groups decided on to accomplish this creative task.

Audience and learning goals.-The choices that the four groups made were shaped by the audience of the problem (i.e., high school students). The groups discussed and made decisions regarding the learning goals for their problem, and expectations of what the targeted students should learn. The learning goals of the problem consisted of enabling secondary school students to utilize their physics knowledge:

\section{Participant $H(G 3):$ The idea is that they would practice with the problem we give them.}

Particularities of the audience whom the problem was addressed to-specifically in terms of their educational level—emerged multiple times; this information was used to assess the conceptual difficulty of the problem, mostly in terms of mathematics representations and concepts. For example,

Participant D (G2): If we think about the students' age, they should know how to carry out such operations.

However, the groups had different focuses. In G1, this discussion concentrated on the reasons to use circular motion as the key concept, and the pedagogical value not only for the targeted students but for themselves as undergraduate students in physics. From the example below, it is possible to perceive the intention of making sense of the activity, its overall objective, and of the principles of circular motion:

Participant A: What is the goal of this (activity)? Of teaching this?

Participant B: What thing?

Participant A: All these equations and concepts.

Participant B: For us or for the students who would be solving this?

Participant A: Well, for both.

Participant B: It is assumed that almost every movement is circular, as it is rare to find truly straight movements. These do not exist. You will see that this (movement) has no angles, when in reality it does.

Participant A: It would be like explaining to them (high school students) how these (movements) work.

The discussion about the learning goal emerged from the task's explicit objective, yet was not necessarily supported by the argument made by participant B in regards to the ever-present circular motion, in an attempt to highlight its importance by transferring the real nature of motion as a combination of circular displacements. The latter argument is interesting, because participants explicitly tried to make sense of the content to be learned by the targeted students, yet this idea received no follow up from participants on G1.

In G2, participants linked the goal of the task to what science teachers would do when facing a similar task. The following segment shows this brief reflection, where the group attempted to convey the appropriateness of their 
problem in coherence with the learning objectives of a physics course:

Participant E (G2): We have to be clear with the goal, which consists of teaching and learning kinematics of circular motion to 12th grade students. So, it is like a teacher getting ready to teach circular motion. That way, each element of circular motion could be linked to different contexts from daily life, or just to one.

This segment provided evidence that, in finding the learning goal, participants mirrored what they thought experts [secondary school teachers] would do in contextualizing the content, and projected their own expectations into what a physics problem should look like. Finally, in G3, we observed a deeper reflection of the learning goal, where a participant highlighted the importance of the reallife context for learning:

Participant $H$ (G3): So, if you include a difficult exercise, but they do not know how to solve it through equations, they might remember that they are addressing a problem that involves a laundry machine where there is a circular motion and they might be able to calculate the speed. Consequently, and lastly, they would understand and know how to calculate angular and tangential speed for a laundry machine, and they would imagine the same type of motion but on different problems.

According to this excerpt, a familiar context would mediate the difficulty of the problem if the targeted students were incapable of applying the required equations, as they would ultimately associate the context of the laundry machine with circular motion. Through this link, participants argued that learners would draw similarities in the use of equations for the purpose of calculating quantities across different scenarios. This, in essence, illustrates a form of knowledge transfer, namely, the use of information from a well-known to an unknown situation. The explicit audience in the task was a key facilitator of this discussion.

Physics concepts and procedures.-With their audience in mind, teams identified, posed, and decided on the physics concepts to use in the problem. In addition, they looked at how these concepts aligned for the generated problem to be well structured, as well as consistent with task requirements. Defining procedures was in direct connection with the learning goal, as teams engaged in the former process to meet the expectations previously defined. When addressing this theme, groups attended to and emphasized different sets of elements, such as the algebraic steps through manipulation of equations, concepts and the combination of quantities for an appropriate problem structure. Figure 2 shows the set of concepts used by each group on their respective problems. For instance, G1 and G2 in Sec. I decided to use the angular version of speed, distance, acceleration as data to determine the magnitudes defined as questions. In contrast, G3 and G4 in Sec. II selected the linear version of speed, distance and acceleration as initial conditions that would allow solvers to determine the number of revolutions completed at the end of motion, the final distance covered, speed, and other questions.

During the early stages of group discussion we observed two different strategies that guided groups in defining their problems: equation driven and concept driven. The first approach (equation driven) was observed in groups that primarily focused on the mathematical resources to support their problem definition, whereas a concept-driven strategy emphasized the conceptual dimension of the situation to then reflect on mathematical representations. G1 mostly utilized an equation-driven approach when defining concepts and procedures. In doing this, they proposed using the equations for "plugging and chugging" the right values in finding the solution:

Participant D (G1): I supposed we need to include the equations. That way we only need to replace what is there. So the problem must be in order. First you calculate one (value), which then allows you to find another.

This process was guided by the (implicit) idea that a problem is constructed in the same way that one may solve it, which refers to following very structured set of steps. This example illustrates the motivation to guide the process of composing the problem by mimicking the algebraic process needed to solve a well-structured physics problem. Similarly, G4 engaged in such a strategy for defining concepts and procedures, yet transitioned towards a concept-driven description of the phenomena after establishing the situation to be used:

Participant M (G4): It will start from rest, and that way we could calculate the movement of the barrel. So then, we would tell them that the barrel is accelerating constantly and that it needs a certain time in seconds to hit the target. Because after some seconds the barrel will be there, at its final position. That is, it will impact the target, so then they could begin their calculations for different things, like angle and everything.

This quote from a member of G4 shows a simple physics analysis of the situation (i.e., a barrel is thrown to a fictional character). Here, the participant analyzed the position and evolution of the object in time, which enabled further understanding of the procedures the targeted students are expected to go through when solving the problem.

Even though deciding on concepts and procedures utilizing a concept-driven approach is not absent from the attention to kinematic equations for problem definition, 
the subtle difference is that the equations emerged after deciding on the concepts. For instance:

Participant E (G2): So let's create a situation where we combine angular speed, acceleration and everything else, like a situation that includes circular distance.

This approach helped the group G2 to create a problem based on the relationship between concepts rather than on the exclusive use of equations. In G3 for instance, participant I attempted to discard the equation-driven approach in favor of using physical interactions (i.e., forces) to describe the phenomena under discussion:

Participant I(G3): More than the equations, it would be better to say that there is a force acting over there, whereas there is another force in that other direction... We need to be more specific. For instance, say that there is a force acting to the inside, and another to the outside.

Even though knowledge about the use of forces goes beyond what the targeted students should master to solve the generated problem, thinking about a qualitative physical description helped the group to build a conceptual framework for the problem.

Besides the different uses of equation- or concept-driven strategy at the early stages of the discussion, all groups moved to the selection of concepts and procedures by combining information available through equations and data created by them (i.e., initial conditions). Because concepts are embedded in the kinematic equations, groups used algebra and physics knowledge to recognize that wellstructured problems must mimic the characteristic of a well-structured system of equations (i.e., number of unknown variables equals the number of known pieces of information). By mimicking this process, group participants utilized variables observed in the equations and made decisions regarding concepts to select as questions, and the needed initial conditions to design a well-structured physics problem. The following interaction from G4 illustrates this process:

Participant N: We could give them the radius for the barrel, and we could also give them a linear speed in $\mathrm{m} /$ $s$, so they have to obtain the angular speed.

Participant L: It would be better if we give them the angular speed and radius, so they can calculate the linear speed.

Participant N: Yes, that works too.

Participant L: And with the linear speed they could then determine how much distance was covered by the barrel since it was tossed.

This negotiation is grounded on the concepts of linear speed $v$, angular speed $\omega$, and radius $R$, and their relationship defined by $v=\omega R$. The discussion then turns into a simple back and forth on whether to use a combination of $v$ and $R$, or rather $\omega$ and $R$, using the missing piece of information as a problem question.

Physics context and wording.-To compose the physics problems, groups also made decisions regarding the contextual elements (i.e., place, subjects, actions etc.) and wording (e.g., A bus is moving from A to B...) of the problem. Groups invested considerable time in doing so ( $21,4 \%$ of the coded data in Table III), which is not surprising taking into account that the activity required them to select a daily situation where circular motion could be easily observed. In general, groups experienced conflict to find the right contextual elements to use because they wanted to engage their audiences. G1 wanted to present a novel situation to the students, so although they started their discussion by only focusing on objects with wheels, they quickly pushed the conversation towards situations without wheels:

Participant D (G1): Is there one without wheels? I cannot think of anything.

On the other hand, G4 wanted to create something fun for the students to be motivated with:

Participant L (G4): Let us do something cool, like a wooden spinning top.

Other participants suggested ideas like a fisherman moving his fishing rod and describing a circular motion, or an ant walking on the inner wall of a bottle. What is important to note is that originality was controlled by their level of confidence in transferring their physics knowledge into such situations, which pushed them to use not only familiar situations, but also situations where they can easily identify, interpret and apply the concept of circular motion. For instance,

Participant D (G1): We do not need to complicate ourselves with that.

As seeing in Fig. 2, G1 selected the wheels of a car moving and covering the distance between two locations; G2 decided on the use of a person trapped in a spinning barrel; G3 decided on the motion of a bicycle; and G4 utilized references from a problem created by their instructor to design a situation where a fictional character tosses a barrel.

The wording of the problems illustrated the use of technical language, and became an opportunity for participants to challenge their own conceptual understandings. A simple example emerged from G1. When deciding on the right wording for the problem:

Participant A (G1): If I want to say that the car wants to move from $A$ to $B$, is this 'displacement'? 
"Displacement" is defined as a vector, so to use it properly the group needed to incorporate a specific direction in the wording of the problem. We found no evidence in the group discussion data of this revision, but their final product (i.e., the problem the group created) was worded using scalars rather than vectors: "a car wants to move" (see Fig. 2).

Another example of discussing an appropriately scientific wording of the generated problem was observed in G4, when participants negotiated the conditions under which the fictional character (Donkey Kong) would make the barrels move:

Participant L: For this, he (Donkey Kong) tosses the barrel from rest?

Participant M: You cannot toss a barrel from rest. It releases the barrel then. He let the barrel go.

Participant L: Ah, okay.

Participant M: Or better, he tosses it with an initial speed, is that okay?

This segment shows the participants' understanding of motion in connection with an appropriate choice of language that conveys the idea that releasing and tossing the barrel implies different physical conditions. Here, a body that begins its motion from rest must be released to accelerate due to the presence of an external force (e.g., gravity), and will therefore gain speed. Differently, tossing implies an interaction (i.e., force) that boosts the kinetic energy and therefore increases the speed to the object in motion.

Discussing magnitudes and units.-Groups engaged in discussions and decision making regarding numerical values and the measurement units of the physics concepts needed to be introduced in the problems' description (e.g., $10 \mathrm{~km} / \mathrm{h}, 20 \mathrm{~s}, 2.5 \mathrm{~km}$ ). Deciding on the numbers and respective units became important because it provided a sense of "reality" to the physical phenomena under design, and therefore imposed requirements over the appropriateness of the data (e.g., initial conditions). The validity of the selected numerical values was tested through the calculation of the questions' response. For instance, G2 discussed the appropriateness of a high angular acceleration for the barrel that yielded to $1400 \mathrm{rpm}$ (revolutions per minute), an unrealistic magnitude that forced them to reduce it. Similarly, in G1, we observed the following interaction when deciding on the acceleration of a car:

Participant A: What do we say the acceleration is?

Participant D: 20.

Participant A: 20 what?

Participant D: Meters by square second.

Participant A: Is that too much?

Participant D: I know it is a lot. Do you want the car to get to its destination fast or slow?
Participant A: I want it to get there at a normal speed.

This dialogue reflects the intention of using numerical values that resemble real life situations. Later on in this process, the same group tested the problem with an acceleration of $10 \mathrm{~m} / \mathrm{s}^{2}$, and got a final speed of $200 \mathrm{~m} / \mathrm{s}$, which is clearly an unrealistic result for a common car moving within city limits.

Brief portions on this topic included data transformation in an effort to add complexity to the problem. Even though the final problem (Fig. 2) shows evidence of this process, the transcripts do not provide much evidence on how these decisions were made. The little evidence shown in the transcripts illustrates the decision of using diameter over radius as a way to add complexity to the problem:

Participant D (G3): Use the diameter so they believe that this is the radius and get all confused.

Or the use of $\mathrm{km}$ instead of $\mathrm{m}$, thus including a data transformation that solvers need to address:

Participant A (G1): Do we include the transformation from kilometers to meters?

Participant D (G1): No, that is not our responsibility.

So far we discussed the findings related to the problem definition category. These included questions about the audience and learning goals; the physics concepts and procedures; the problem context and the wording; and the magnitudes and units used. In the next section, we develop the other important category that emerged from the data, the problem-solving strategies. In this other category, we found processes related to physics and mathematicsspecific concepts and representations, and typically related to solving well-structured problems. Within this category, two main themes were discussed: algebraic procedures and steps; and physics of circular motion.

\section{Problem-solving strategies}

The following set of themes emerged from participants engaging in processes often associated with solving wellstructured physics problems, where participants were likely to utilize physics concepts and their mathematical representations, while engaging in physics descriptions linked with the context of the activity. The literature on both novice and expert physics problem solvers suggests that novices tend to utilize algebra-based strategies (e.g., plugand-chug) rather than qualitative descriptions, a strategy associated with expertise $[23,24]$.

Algebraic procedures.-This process relates to the algebraic steps that the group engaged in to obtain the numerical values needed to test the appropriateness of their problems. 
Here, we found that they used their mathematical knowledge as a strategy to determine the correct physics quantity:

Participant A (G1): Here we will use a proportionality rule. If one revolution is $2 \pi$, then $x$ revolutions will be...

And/or asked for help on how to proceed in order to get the right value:

Participant $F$ (G2): How do we transform this to radians? Does someone know how to do it?

To contextualize the use of algebra in this context, it is important to remember that kinematics problems rely on three fundamental physical quantities: position $[\vec{r}(t)]$, velocity $[\vec{v}(t)]$, and acceleration $[\vec{a}(t)]$, all functions of time $t$. Even though these concepts are defined as vectors, in this context participants utilized them to determine scalar quantities, or the magnitudes of the vectors at any given time. In circular motion, these concepts are written in an angular form: angular position $[\theta(t)]$, angular speed $[\omega(t)]$, and angular acceleration $[\alpha(t)]$. The link between these linear and angular magnitudes comes from $s=\theta R$ (i.e., $\operatorname{arc}), v=\omega R$, and $a=\alpha R$. Consequently, in order to test the appropriateness of their problems, participants manipulated some or all of the latter mathematical relations. For instance, participants in G4 had the following argument to determine the angular distance covered by the barrel:

Participant L (G4): And how would I get the angle?

Participant M: With the (angular) acceleration that is obtained from the equation. With the angular speed. Because you have the angular speed at 3 s, which is $10 \pi$, so $10 \pi$ is equal to (angular) acceleration plus the initial angular speed. So then you freed it and get the (angular) acceleration.

Here, participant $\mathrm{M}$ suggested the use of angular speed $[\omega(t)]$ at time $3 \mathrm{~s}$, to determine the value of angular acceleration by isolating this from the equation, because all other elements were given. Then, although not explicitly mentioned, the argument oriented the use of the numerical value of angular acceleration into the equation for angular position, to determine $\theta$ at $3 \mathrm{~s}$. Once again, this was possible because all of the other elements of the equation were defined.

Another interesting example was observed in G1, as they used the equations $\vec{r}(t)=\vec{r}_{0}+\vec{v}_{0} t+(1 / 2) \vec{a} t^{2}$ and $\vec{v}(t)=$ $\vec{v}_{0}+\vec{a} t$ to determine the time that it would take the car to reach its destination. The following interaction depicts the set of algebraic steps suggested by one participant to achieve this goal:

Participant A (G1): So we have that the initial position is zero, and the initial speed is zero. And we have that (in the equation), only the acceleration times squared time will remain.
Participant B (G1): And then?

Participant A: That will give you $10 \mathrm{~m} / \mathrm{s}^{2}$ (magnitude of the acceleration) multiplied by the squared time, and with that you can get the time. So, it will be the square root of something, and then we will use only the positive square root.

This interaction shows a description about how to manipulate the terms in the equation $\vec{r}(t)=\vec{r}_{0}+\vec{v}_{0} t+$ $(1 / 2) \vec{a} t^{2}$ to determine the time, provided that all the other terms are known (final distance was given in the heading of the problem and was equal to $2 \mathrm{~km}$, Fig. 2). Because this algebraic procedure might have been perceived as a rather individual exercise, it is not surprising that the audio only captured brief descriptions of this strategy implemented to find numerical values, and the request for help on how to calculate them.

Physics of circular motion in context.--Under this strategy, groups addressed qualitative physics descriptions of the context under consideration for the problems they were designing. This process enabled access to participants' conceptualization of the physics phenomena and the ways in which they would explain such situations in physical terms. The sample size of examples that illustrates this process is rather small and does not provide evidence of how students explain most of the concepts. Consequently, the observed frequency of this theme (see Table III) might reflect the disparity between algebraic-versus-qualitative strategies shown by participants. Moreover, qualitative descriptions emerged from the data when participants tried to make sense of the situations and physical objects considered for the problem.

Participants attempted to explain revolutions, tangential velocity, and inertia. The latter was introduced to explain what would happen if someone were to fall from a fastspinning carousel. For instance, the concept of a revolution was conceptualized through the perimeter of a wheel in a simple description that does not reflect a deep understanding of a physics concept associated with motion:

Participant A (G1): Suppose that first there is a point that moves along the perimeter until here. That will be one revolution.

A more interesting example was provided by $\mathrm{G} 2$ in an attempt to understand the relationship between angular and linear speed in the context of a wheel moving. First, angular speed is defined as the change of angular position per unit of time (i.e., $\omega=\Delta \theta / \Delta t$ ), a magnitude difficult to conceptualize because it does not include distance units, such as meters or kilometers. Secondly, an object spinning will measure the same angular speed at any distance from the center of rotation (i.e., radius) at a particular time. However, the linear or tangential speed will increase according to the distance from the center of rotation as 
shown by the equation $(v=\omega R)$. The discussion then unfolded as follows:

Participant E: If this is supposed to be in the same wheel, then why? If you advance five meters, you will complete the same number of revolutions.

Participant G: Yes, you are right. So, how many...

Participant F: The angular speed will change at different points of the wheel.

Here we see that participant $\mathrm{F}$ poses a misconception regarding the nature of angular speed, because this quantity remains constant regardless of the distance from the center of the rotating object. Consistent with the definition of angular speed, the comment made by participant $\mathrm{E}$ would have made more sense if instead of using $5 \mathrm{~m}$ as the distance covered, he would have used angular measurements to highlight the distinction between angular and linear speed.

In a last example, we observe a qualitative description from G3 when participants discussed the relationship between force and acceleration in circular motion:

Participant I: There is also velocity, this velocity that goes to the middle. This is the one that enables... This was related to forces if I remember. The topic of the two forces pointing out to one side. Now I remember, centripetal and centrifugal force. Centrifugal force was like...

Participant H: It is the one that points inside.

Participant I: No.

Participant J: Centripetal force points to the inside.

Participant I: Okay, centripetal force points to the inside.

But centrifugal points to the outside. And those two forces would make that...There were like equals and...

Participant H: Both forces allow the circular motion.

Participant I: But this centrifugal force was something

like hypothetical, or something that was not real...

This interaction shows that participants made an effort to understand the physical interactions that enable circular motion. Their ideas about centripetal force are correct: it is an interaction (force) directed to the center of the circumference described by the body in motion, and responsible for such motion. However, centrifugal force, as corrected by participant I at the end of the interaction, is not a force but rather the effect of inertia, defined as resistance to change the state of motion, and often referred to as a "fictitious force." Finally, this interaction provides some insights on participants' understanding, but again falls short to give substantial evidence to assess whether participants were actually understanding the underlying physics of circular motion beyond the use of equations.

As developed in the present subsection, the majority of the group discussions revolved around the problem definition and problem-solving strategies. However, the groups invested their efforts differently in every stage of the task.
In the next subsection we present and compare the frequency of coded themes among groups to show what processes demanded more or less group negotiation.

\section{B. Group comparison}

To illustrate how groups combine their efforts for composing a physics problem, Fig. 3 depicts the percentage of coded themes by group. The black dotted line represents the average frequency.

A simple scanning of Fig. 3 shows that problem definition processes demanded higher investment than problem-solving strategies across all four groups. Within the first category (problem definition), groups displayed similar engagement when discussing the learning goals. Further, most of the ideas coded were related to physics concepts and procedures. Here, both G1 and G2 invested about the same percentage deciding on the physics (approximately $35.5 \%$ ), whereas G4 invested $10 \%$ more on this process $(45.7 \%)$. Further, deciding on the context of the problem is one of the categories where we can observe most disparity across groups, with G3 and G4 (Sec. II) engaging in this process consistently more than G1 and G2 (Sec. I). For magnitudes and units, G2 and G3 were the ones that spent close to $20 \%$ of their group processes to discuss and agree on these topics.

The algebraic procedures show diverse engagement, with only G1 scoring above average. When it comes to qualitative descriptions of the physics in context, all four groups show a similar trend for algebraic procedures, with the exception of G2, a team that only engaged in this process $2.1 \%$ of their coded data. Finally, we must be cautious when interpreting this evidence because students may have engaged in these processes silently. Consequently, it might be possible that students in G1 verbalized their mathematical procedures at a higher rate compared to the rest of the sample.

\section{Benefits, challenges, and collaboration}

While the previous section addressed research question 1 related to the physics and ideas that groups focused on when solving the task, this last subsection provides evidence regarding research questions 2 and 3:

RQ2: What are the perceived benefits and challenges of composing a physics problem for high school students?

RQ3: How do participant groups collaborate when composing a physics problem for high school students?

We begin by showing evidence associated with the benefits and challenges of composing physics problems, and then we move to the ways in which the participant groups collaborated and interacted during this activity. In each of these subsections we identify attributes and strategies for solving traditional well-structured physics problems and the real-world problem. Here, we first describe problem features and challenges by making a parallel between these two types of learning activities. 


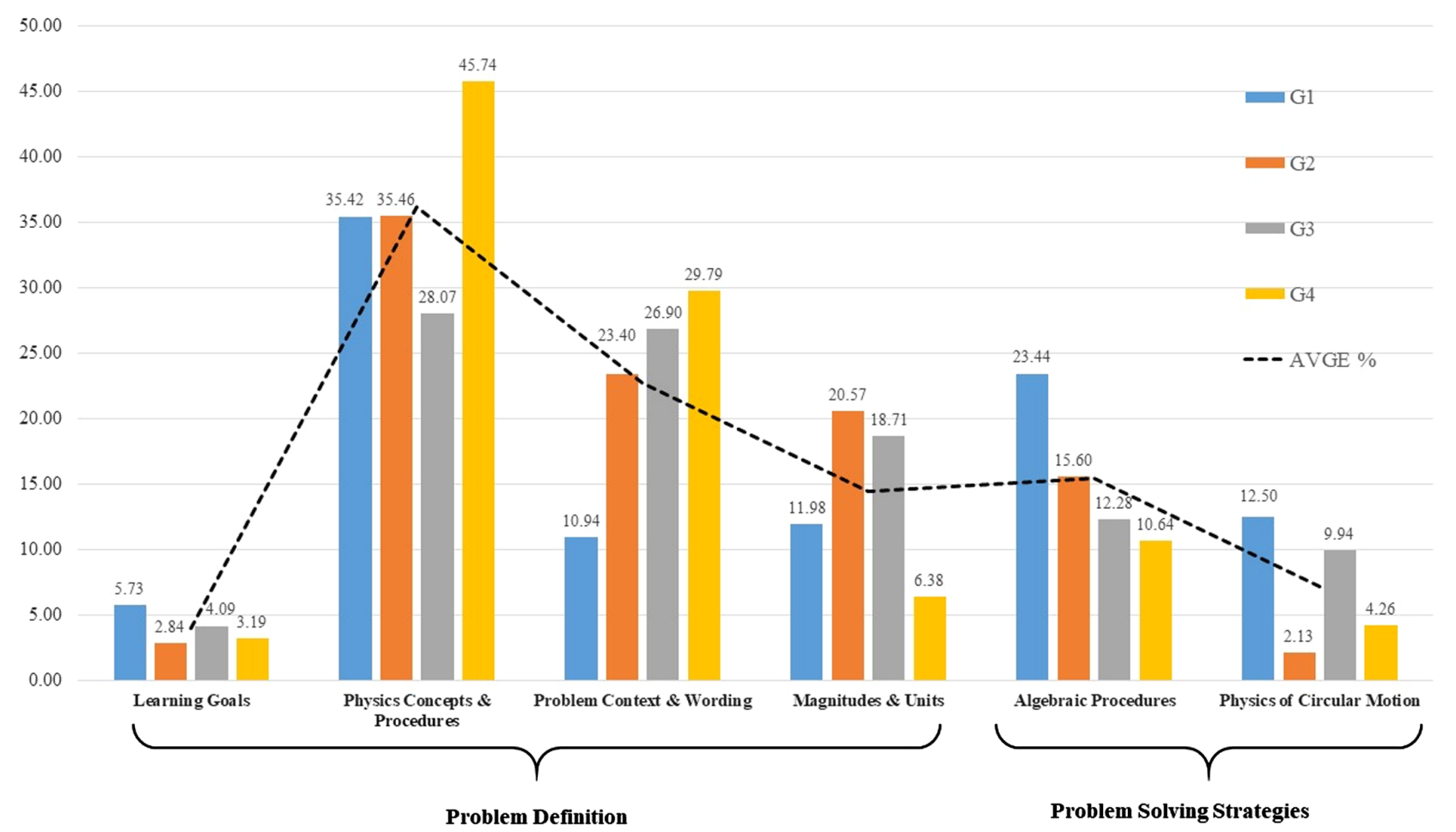

FIG. 3. Percentage of coded themes by group. Dotted line represents the average percentage across the four groups.

Second, we address the ways in which students interact with others (i.e., collaboration strategies) when facing wellstructured problems and real-world problems. Finally, through this description we refer to questions from the semistructured interview protocol available in the Supplemental Material [61], and the follow-up questions that came up during the interviews.

\section{Benefits and challenges of composing a physics problem}

Through participants' interviews we found unique sets of attributes, challenges, and strategies associated with either traditional well-structured problems and real-world problems. Consistent with the documented limitations of these problems for conceptual development, when we asked participants about engaging in qualitative analysis of physics principles (Interviewer: "When solving well-structured problems, do you take the time to reflect on the underlying physics?"), they claimed that they discarded such conceptual considerations because the goal was to find the appropriate solution. For instance,

Participant B $(G 1)$ : When I solve it do not reflect on it.

Similarly,

Participant G (G2): Generally and above all we need the formulas to find the value that they are asking for, for example velocity.
Although these answers can indicate that participants frequently engage in novice problem-solving strategies, one participant acknowledged the value of well-structured problems as a way of knowing the physics concepts related to the problem:

\section{Participant B (G1): The well-structured problem allows you to know the concepts.}

Conversely, when it comes to composing a physics problem (Question 5, interview protocol), participants suggested that the most challenging elements of this activity were associated with imagination and making appropriate decisions for the problem. For Participant B (G1) their lack of familiarity with processes related to idea-generation and decision-making made it difficult to move forward:

\section{Participant B (G1): It is harder because we are not used to creating our own problems.}

Participant J (G3) compared this with the characteristics of well-structured problems and declared

Participant $J$ (G3): Obviously it is more difficult (composing a problem) because we have to take in consideration many things that are present in wellstructured problems.

Among these missing elements, participants pointed out their struggles towards finding real-life magnitudes and units to compose realistic scenarios for their problems. 
Moreover, participants recognized that the latter challenges afforded them learning opportunities (Question 6, interview protocol). For participant $\mathrm{G}(\mathrm{G} 2)$, having an appropriate understanding of the physics concepts and principles facilitated decision-making over magnitudes and units:

Participant $G(G 2):$ They ask you to make a realistic problem, so you have to think about that, and your results must be things that exist. You are not going to define an airplane moving at $5 \mathrm{~m} / \mathrm{s}$. So it forces you to understand the content better.

Similarly, the same participant highlighted the importance of understanding the content to find the appropriate complexity for the problem:

Participant G: When you do not know the content well one has the tendency to provide more information than necessary, because you do not know whether you would get to the expected result. So you add more information to the problem, and with this extra information the problem gets undesirably easier.

Accordingly, the amount of information provided in the problem must align with its complexity, thus reflecting on how well participants understood physics concepts and its relations to compose a problem.

Furthermore, when the interviewer asked participants whether they reflected on the underlying physics for composing a problem, the open-ended nature of the task was perceived as an opportunity to reflect on physics content by contextualizing their ideas into real-life scenarios. When composing a physics activity, participants considered the underlying concepts differently. For instance,

Participant B (G1): Composing a problem motivated me think not on an ideal concept, but a real one. So I think of friction that a given character must experience with the wind.

Participant G (G2) underscored the importance of understanding how physics concepts work in context:

Participant G(G2): Because circular motion works like this, and you see it on a wheel because the wheel is spinning, and also see that the tangential motion is related to the spinning of the wheel while moving on the ground.

In a similar fashion, Participant L (G4) suggested that composing a physics problem makes you perceive daily life activities through physics knowledge:

Participant L: The other day they taught us relative motion, and I was on the bus and began thinking about it."
Finally, Participant J (G3) went beyond the boundaries of the physics classroom and found similarities between composing a problem for physics and engaging on an engineering project:

Participant J (G3): We had to design a vehicle and I had never seen mechanics. I studied a little in school, but a different thing is to transfer it into real life instead of doing it on a piece of paper. It is very different and obviously one gains more by doing it in real life.

Then, the participant acknowledged the value of solving a real-world problem, because it positions her closer to what she would end up doing in a few years as an engineer. Accordingly, and similar to participant B, both recognized the value of getting used to actively making decisions based on their knowledge, as it was considered a key ability in the face of real-world problems.

\section{Collaboration strategies when composing a physics problem}

Participants engaged in different collaboration strategies depending on the nature of the activity. A general consensus was found across interviewees that when groups faced well-structured physics problems they embraced individualistic problem solving and interacted with each other to overcome emerging difficulties and/or compare their results. For instance, as a response to the group processes solving well-structured problems (Question 8, interview protocol), Participant J (G3) claimed

Participant $J(G 3)$ : We first read all the problems, and then we identify the data available. If there are three questions, we divide the questions. Then we begin on our own, and then we obviously ask about what can I do with this?

Consequently, for participant J (G3) within group interactions takes place at the beginning and/or end of the problem-solving process, where the group requests factual information (e.g., "What formula did you use?"). Furthermore, participants recognized that if their group knowledge and skills were not enough, they resorted to seeking out information to other groups, a form of collaboration that was possible because the classroom was working on the same problems. Pursuing factual information for solving a well-structured physics problems was, presumably, what drove social interactions within and between groups, as it seemed that the main goal was to find the right answer:

Participant B (G1): When I search for information I do not pay attention to the why, but rather on the result and finish the exercise.

In response to collaboration when composing a physics problem (Question 8, interview protocol), participants 
claimed that their ideas came up after brainstorming and collective decision making. Participant B (G1) described this cooperation as:

Participant B (G1): We were talking and sharing ideas and suddenly we had it (a good idea), and we began to identify what we needed to make it attractive for the kids (high school students).

From the complexity of the assignment participants recognized the importance of working with each other rather than individually. The same participant B (G1) stressed out the importance of collaboration in this scenario:

Participant B (G1): We all have to pay attention or we may miss something.

Similarly, and contrasting with solving a well-structured problem:

Participant $J(G 3)$ : We read the problem and said that we need to work on this together, so we began to discuss ideas.

These testimonies suggest that composing a physics problem demands higher levels of positive interdependence, a necessary condition for cooperative work.

Moreover, reaching out to other groups for information was the only evidence between sections that could be attributed to the different strategies in which instructors guided the problem-solving session. As a reminder, the instructor in Sec. I (G1 and G2) motivated social interactions by guiding participants to seek out information from their peers, and the instructor from Sec. II (G3 and G4) provided direct information to participants' inquiries. It seems that group interactions were a consequence of a particular instructional strategy, as groups from Sec. I sought out information from other groups, while groups 3 and 4 did not. In G1, participant B mentioned that they reached out to other groups to get "an alternative point of view," while participant G (G2) mentioned having "heard a good idea outside their group." Conversely, for participants J (G3) seeking out information to other groups was not necessary, because

Participant $J(G 3)$ : Each group would do the things that they liked, and it is quite subjective.

Similarly, participant K (G4) suggested

Participant $K(G 4)$ : Because all problems are different, I do not need to compare the answer with other groups, as these could be completely different.

Further explanations on whether students took advantage of information from other groups or not are presented later in the discussion section.
Finally, interesting evidence emerged on the nature of the information that participants sought to compose the physics problem (Question 9, interview protocol). If students tended to pursue factual information to solve a wellstructured problem, then a creative activity would motivate them to ask procedural questions to understand how to apply the physics concepts into the problem. This shift was identified in the data. For instance,

Participant B (G1): I try to be more thorough with my questions. For example, how did you do this? And why did you do it? I ask them everything.

This evidence is coherent with the demands that composing physics problems impose; this task directs students to address the conceptual dimension of the content and ultimately shapes the types questions and interactions that participants engage in.

\section{DISCUSSION}

Frequently composing physics problems is a responsibility taken up by physics educators rather than undergraduate students. However, in this study we explored the learning advantages and challenges of incorporating this activity in an undergraduate curriculum. The complexity of composing a physics problem requires making choices over multiple related dimensions, depending on the nature of the problem at hand [12,40]. From the analysis of groups' recordings we found that participants' discussions revolved around problem definition and problem-solving strategies. In the former category, we identified the following themes: 1. audience and learning goals; 2. physics concepts and procedures; 3 . problem context and wording; 4. magnitudes and units, and in the second category, problem-solving strategies, involved 1. algebraic procedures; and 2. physics of circular motion. According to Fortus [12], open-ended physics problems require making choices about two different but related issues, such as physics variables and principles, and the magnitudes of these variables. The first set of assumptions are accessible to both novices and experts, mainly because both levels of expertise have been exposed to physics concepts and principles to some extent. This exposure in turn grants them the mastery to decide on the set of concepts to manipulate and how to do it. However, assumptions regarding the numerical magnitudes of the variables used in the problem has been found to be harder for novices (e.g., undergraduate students) compared to expert problem solvers (e.g., graduate students) [12], presumably a consequence of their lack of familiarity in manipulating information in such form. The analysis of group discussions and interviews supports this claim, as participants struggled to identify numerical values that would mimic real life situations in their composed problems. 
In relation to the difficulties experienced by novices and experts when addressing physics concepts and procedures and magnitudes and units, we argue that choices about the problem context and wording are more familiar to both novices and experts. Here, both novices and experts are likely to have experience reading different types of wellstructured physics problems, with various contextual details and wording. Therefore, participants may be more trained in using that knowledge as a resource to make their own choices. In contrast, and even though all participants might have been exposed to learning activities of a diverse nature, discussing and making decisions about the audience and learning goals may constitute a more challenging endeavor, as it entails knowledge of the targeted students which would ultimately mediate the problem's difficulty. From this analysis, we found that the activity of composing problems can add two alternative types of assumptions with arguably different levels of complexity for both experienced and nonexperienced solvers: problem context and wording; and audience and learning goals. We deem this as relevant for students to develop familiarity with cognitively demanding processes of knowledge transfer and decision making [29].

We know from a writing studies perspective that genres constitute responses to previous genres [65]; consequently, student writing is generally a response to a prompt-an instructor's genre. The findings in this study underscore the role that the prompt has in guiding students' thinking and decision-making processes. As a WTL task, the prompt provided the participants with a clearly articulated rhetorical context, mainly by making explicit the audience of the problem, the genre, the purpose and the role of the writers [66]. The data show that this information offered a road map for the participants to create the problem and to discuss choices. In all cases, the participants relied on the information about the audience (high school students) to select concepts, to establish the degree of complexity of those concepts, and to select rhetorical and other devices that would engage the audience. In a study of 2101 writing assignments across the curriculum in 100 U.S. higher education institutions, Melzer [66] found that $64 \%$ of writing assignments did not clearly state an audience, and that in most cases the audience was the teacher in the role of an examiner. The absence of an audience, according to the author, limits the pedagogical possibilities of an activity. Our study extends Melzer's finding about the role of the audience in undergraduate assignments, and provides evidence of the specific disciplinary choices and discussions that are introduced into an activity when the students know whom they should address with their writing.

Other characteristics of this WTL task that were central to scaffold our participants' composing processes were the explicit reference to the genre (physics problem) and the role as writers (university community members). In both the group recordings and interviews, participants referred to different characteristics of expected genre to make particular choices. It was mainly in the interviews where we could identify how our participants' past experiences with the genre physics problem informed their decisions. Additionally, the task positioned the participants in a more expert role, one that demanded them to negotiate and make decisions. This fostered discussions about the choices, frequently supported by explanations and reasons of the disciplinary logic behind their thoughts. For example, the participants enacted a concept-driven approach to decide what relevant physics information to include in their learning activities, a process that physics instructors frequently engage in. Lastly, the specific content of the discussions indicate that the activity promoted agency, because through the decision-making process participants displayed control over the meanings that they wanted to convey [67], as well as metacognition, since participants had to articulate the reasons behind their choices [68]. Overall, the rhetorical challenges of this WTL task and its collaborative component guided participants into performing disciplinary thinking and reasoning, experiences that can potentially lead to more sophisticated understandings about the subject matter [19].

In addition, results show that composing physics problems encouraged participants to engage in both quantitative (algebraic procedures) and qualitative (physics of circular motion) strategies for solving their problems. It is important to point out that the frequency of coding and group comparison indicated different investment on both of these problem-solving strategies. Participants' discussions were mostly about algebraic procedures rather than qualitative descriptions. Yet, as seen from the analysis, this qualitative description favored learning opportunities through peer regulation. For instance, evidence shows how participants addressed and discussed the nature of circular motion by centripetal and centrifugal forces, but tackled the misconception of the latter as a real interaction, a common conceptual challenge in circular motion [45,47].

The PER literature has recognized the challenges of finding a balance between both problem-solving strategies for encouraging physics expertise [2,69]. Composing problems might guide students in the right direction, but further analysis and experimentation are required. For instance, it could be useful to think about using characteristics from isomorphic sets of physics problems [22,23] in order to encourage participants to compose pairs of quantitative and conceptual problems. Here, generating both quantitative and qualitative physics problems that deal with the same content may increase the reflection of physics related ideas beyond the sole utilization of mathematical representations.

The ways in which participant groups collaborate for solving well-structured and real-world problems is relevant in order to understand the social processes that govern each 
of these activities. Participants' interviews suggest that when solving well-structured physics problems, students first enacted individual work, as every group member attempted to solve the problem. Further interactions within the group might occur to overcome difficulties and/or test results. It is already known that when solving wellstructured problems, or disjunctive tasks [70], students do not engage in group-level processes [5]. In addition, this collaborative strategy is enacted to pursue factual and simple information related to the problem's solution, thus mirroring problem-solving strategies often engaged by novices [22,23]. Differently, and even though we did not look for team-related processes throughout the data, participants' interviews pointed towards collective decision making when composing the physics problem, as well as the underlying reasons for using a given concept and procedure. The nature of such a collective process is evidence of the additive nature of the task [7] and its required positive interdependence [5] that motivates the understanding of the procedures and knowledge used by others, a strategy that resembles expertlike behavior $[20,21]$.

The nature of the group interactions when composing a problem might be shaped by the type of instructional guidance as well as behavioral expectations. The interviews suggested that interactions among groups tended to occur in the context of well-structured problems, and for similar purposes than the interactions within groups (i.e., to overcome difficulties and/or test results). This might have been due to the task shared goal across the course. Yet, interactions occurred differently when composing physics problems: participants from Sec. I mentioned the strategy of reaching out for information to other groups, while participants from Sec. II claimed the lack of a common goal between groups as the reason why they did not follow such a route. The contrasting strategies between sections might be attributed to the different scaffolding provided by the instructors. The instructor in Sec. I guided participants to seek out information from other classroom participants during the problem-solving session, whereas the instructor in Sec. II provided direct responses to participants' questions. More research is needed on this topic to expand this idea. There is still a great gap in the knowledge of the implications and benefits for student groups when composing physics problems and other real-world activities. Further PER research could explore, for instance, whether instructional strategies influence how students collaborate for creative tasks, or whether the group configuration in terms of gender, achievement and other attributes contributes to their performance. Both suggestions could potentially provide guidelines for implementing collaborative activities. This research avenue is timely with the current demands of the XXI century skills of creativity and collaboration [39,71], a great motivation for educators and scholars to engage in pedagogical innovations.

\section{CONCLUSIONS}

Lately, the PER community has provided different types of evidence to show the pedagogical benefits of incorporating real-world problems in the physics classroom. The current study built on this approach and extended what was already known about the subject by exploring the process of solving real-world problems from the students' perspective and incorporating a WTL approach.

The findings from this study not only point to the advantages of incorporating real-world problems in the physics curriculum, but also to the role that well-defined writing tasks play in scaffolding the process (i.e., those that incorporate explicit goals, audiences, steps, and expectations). When our participants were presented with the task of writing a physics problem for a specific audience and context, they had to make choices over concepts, degree of complexity, and goals. The audience and the context were central to scaffold the students' thought processes, since the majority of the choices made by students were discussed in these terms. More importantly, the additive nature of composing problems motivates the access to alternative ideas through deeper questioning, among which we observed processes typically engaged in solving traditional physics problems.

Collaboration allowed students to negotiate, address and make decisions regarding a multiplicity of choices, and it fostered the process of idea generation. This form of student collaboration contrasts with the simple ways in which participants described interactions for solving wellstructured physics problems, as they claimed to seek factual information rather than more complex insights. Interestingly, instructors could foster collaboration beyond what one would typically observe in the form of within-group collaboration, and particularly for learning tasks with outcomes expected to be different across groups. This implies that instructors have alternatives to frame and model the type of interactions desired for the class. Whether it is by fostering within-group interactions or across group collaboration, it should respond to academic and disciplinary expectations made explicit through instruction.

This study has pedagogical implications as well. When incorporating writing activities in the physics curriculum, the activity must make the genre explicit, establish a concrete audience and context, as well as its goals and expectations. Preferably, the task should enable students to connect their personal experiences with the disciplinary knowledge. Educators and researchers could draw on the analyzed categories and themes to assess and/or investigate students' work on similar activities. Such guidelines could also be helpful if made explicit to students, and could be used by them to regulate their performance and interactions. 


\section{ACKNOWLEDGMENTS}

This material is based upon work supported by the AAPT E. Leonard Jossem International Education Fund. Any opinions, findings, and conclusions or recommendations expressed in this material are those of the author(s) and do not necessarily reflect the views of the American Association of Physics Teachers. We acknowledge and appreciate the work of Carlos Rios Morales in facilitating the research setting for conducting this study.
[1] E. Kim and S. Pak, Students do not overcome conceptual difficulties after solving 1000 traditional problems, Am. J. Phys. 70, 759 (2002).

[2] T. Byun and G. Lee, Why students still can't solve physics problems after solving over 2000 problems, Am. J. Phys. 82, 906 (2014).

[3] P. Heller and M. Hollabaugh, Teaching problem solving through cooperative grouping. part 2: Designing problems and structuring groups, Am. J. Phys. 60, 637 (1992).

[4] P. Heller, R. Keith, and S. Anderson, Teaching problem solving through cooperative grouping. part 1: Groups versus individual problem solving, Am. J. Phys. 60, 627 (1992).

[5] D. W. Johnson, R. T. Johnson, and E. J. Holubec, Circles of Learning: Cooperation in the Classroom (Interaction, Edina, MN, 1986).

[6] D. H. Jonassen, Toward a design theory of problem solving, Educ. Technol. Res. Dev. 48, 63 (2000).

[7] I. D. Steiner, Models for inferring relationships between group size and potential productivity, Behavioral Science 11, 273 (1966).

[8] J. Pulgar, C. Candia, and P. Leonardi, Social networks and academic performance in physics: Undergraduate cooperation enhances ill-structured problem elaboration and inhibits well-structured problem solving, Phys. Rev. Phys. Educ. Res. 16, 010137 (2020).

[9] R. Teodorescu, C. Bennhold, G. Feldman, and L. Medsker, New approach to analyzing physics problems: Ataxonomy of introductory physics problems, Phys. Rev. Phys. Educ. Res. 9, 010103 (2013).

[10] D. P. Maloney, An Overview of Physics Education Research on Problem Solving (American Association of Physics Teachers, College Park, MD, 2011).

[11] N. Shin, D. H. Jonassen, and S. McGee, Predictors of wellstructured and ill-structured problems solving in astronomy simulation, J. Res. Sci. Teach. 40, 6 (2003).

[12] D. Fortus, The importance of learning to make assumptions, Sci. Educ. 93, 86 (2008).

[13] J. Lave and E. Wenger, Situated Learning: Legitimate Peripheral Participation (Cambridge University Press, Cambridge, England, 1991).

[14] E. Brewe, L. H. Kramer, and G. E. O'Brien, Changing participation through the formation of student learning communities, AIP Conf. Proc. 1289, 85 (2010).

[15] V. Tinto, Classrooms as communities: Exploring the educational character of student persistence, J. Higher Educ. 68, 599 (1997).
[16] A. J. Herrington and M. Curtis, Persons in Process: Four Stories of Writing and Personal Development in College. Refiguring English Studies Series (National Council of Teachers of English, Urbana, IL, 2000).

[17] K. Roozen, Coming to act with tables: Tracing the laminated trajectories of an engineer-in-the-making, Learning Cult. Social Interact. 24, 100284 (2020).

[18] A. R. Gere, N. Limlamai, K. Wilson, E. MacDougall Saylor, and R. Pugh, Writing and conceptual learning in science: An analysis of assignments, Written Commun. 36, 99 (2019).

[19] J. Meyer and R. Land, Overcoming Barriers to Student Understanding: Threshold Concepts and Troublesome Knowledge (Routledge, London, New York, 2006).

[20] J. Larkin, J. McDermott, D. P. Simon, and H. A. Simon, Expert and novice performance in solving physics problems, Science 208, 1335 (1980).

[21] R. J. Dufresne, W. J. Gerace, P. T. Hardiman, and J. P. Mestre, Constraining novices to perform expert-like problem analyses: Effects on schema acquisition, J. Learn. Sci. 2, 307 (1992).

[22] D. Meltzer, Relation between students' problem-solving performance and representational format, Am. J. Phys. 73, 463 (2005).

[23] C. Shing, Assessing student expertise in introductory physics with isomorphic problems. II. Effect on some potential factor on problem solving and transfer, Phys. Rev. Phys. Educ. Res. 4, 010105 (2008).

[24] J. L. Docktor, N. E. Strand, J. P. Mestre, and B. H. Ross, Conceptual problem solving in high school physics, Phys. Rev. Phys. Educ. Res. 11, 020106 (2015).

[25] W. J. Leonard, R. J. Dufresne, and J. P. Mestre, Using qualitative problem-solving strategies to highlight the role of conceptual knowledge in solving problems, Am. J. Phys. 64, 1495 (1996).

[26] L. Buteler and E. Coleoni, Solving problems to learn concepts, how does it happen? A case for buoyancy, Phys. Rev. Phys. Educ. Res. 12, 020144 (2016).

[27] R. Leinonen, M. A. Asikainen, and P. E. Hirvonen, Peer discussion in lectured-based tutorials in introductory physics, Phys. Rev. Phys. Educ. Res. 13, 010114 (2017).

[28] J. Hardy, S. P. Bates, M. M. Casey, K. W. Galloway, R. K. Galloway, A. E. Kay, P. Kirsop, and H. A. McQueen, Student-generated content: Enhancing learning through sharing multiple-choice questions, Int. J. Sci. Educ. 36, 2180 (2014). 
[29] J. P. Mestre, Probing adults' conceptual understanding and transfer via problem posing, Appl. Dev. Psychol. 23, 9 (2002).

[30] H. Estrem, Disciplinary and professional identities are constructed through writing, in Naming What We Know: Threshold Concepts of Writing Studies, edited by L. Adler-Kassner and E. Wardle (University Press of Colorado, Boulder, CO, 2015).

[31] C. Bazerman, J. Little, and L. Bethel, Reference Guide to Writing Across the Curriculum (Parlor Press LLC, West Lafayette, Ind., 2005).

[32] C. R. Miller, Genre as social action, Quart. J. Speech 70, 151 (1984).

[33] J. Pulgar, A. Spina, S. Ríos, and D. Harlow, Contextual details, cognitive demand and kinematic concepts: exploring concepts and characteristics of student-generated problems in a university physics course, in Proceedings of 2019 Physics Education Research Conference, Provo, UT(AIP, New York, 2019).

[34] C. Bazerman, A Theory of Literate Action: Literate Action Volume 2. Perspectives on Writing (The WAC Clearinghouse, Parlor Press, Anderson, South Carolina, 2013).

[35] J. Hoehn and H. J. Lewandowski, Framework of goals for writing in physics lab classes, Phys. Rev. Phys. Educ. Res. 16, 010125 (2020).

[36] C. Bereiter and M. Scardemalia, The Psychology of Written Communication (Lawrence Erlbaum, Hillsdale, NJ, 1987).

[37] K. Quillin and S. Thomas, Drawing-to-learn: A framework for using drawings to promote model-based reasoning in biology, CBE Life Sci. Educ. 14, es2 (2015).

[38] D. K. Riser, S. D. Clarke, and A. N. Stallworth, Scientific memes: Using the language of social media to improve scientific literacy and communication in lifespan development, Psychol. Learning Teaching 19, 275 (2020).

[39] S. Pelger, Popular science writing bringing new perspectives into science students' theses, Int. J. Sci. Educ. Part B 8, 1 (2018).

[40] W. Rietman, Heuristic Decision Procedures, Open Constraints, and the Structure of Ill-Defined Problems (Wiley, New York, 1964).

[41] R. J. Marzano and S. Kendall, The New Taxonomy of Educational Objectives, 2nd ed. (Corwin Press, Thousand Oaks, CA, 2007).

[42] C. K. W. De Dreu, B. A. Nijstad, M. N. Bechtold, and M. Bass, Group creativity and innovation: a motivated information processing perspective, Psychol. Aesth. Creativity and Arts 5, 81 (2011).

[43] R. K. Sawyer and S. DeZutter, Distributed creativity: how collective creations emerge from collaboration, Creativity and Arts 3, 81 (2009).

[44] T Amabile, Creativity in Context (Westview Press, Boulder, CO, 1996).

[45] D. Hestenes, M. Wells, and G. Swackhamer, Force Concept Inventory, Phys. Teach. 30, 141 (1992).

[46] J. Roche, Introducing motion in a circle, Phys. Educ. 36, 399 (2001).

[47] A. Volfson, H. Eshach, and Y. Ben-Abu, Identifying physics misconceptions at the circus: The case of circular motion, Phys. Rev. Phys. Educ. Res. 16, 010134 (2020).

[48] L.S. Vygotsky, Mind in Society: The Development of Higher Psychological Processes (Harvard University Press, Cambridge, MA, 1978).

[49] J. J. B. Harlow, D. M. Harrison, and A. Meyertholen, Effective student teams for collaborative learning in an introductory university physics course, Phys. Rev. Phys. Educ. Res. 12, 010138 (2016).

[50] M. Eodice, A. E. Geller, and N. Lerner, The Meaningful Writing Project: Learning, Teaching and Writing in Higher Education (University Press of Colorado, Boulder, CO, 2016).

[51] R. K. Sawyer, Group Creativity, Music, Theater, Collaboration (Lawrence Erlbaum Associates, Publishers, London, 2003).

[52] J. Baruah and P. B. Paulus, Effects of training on idea generation in groups, Small Res. Group 39, 523 (2008).

[53] L. Thompson, Improving the creativity of organizational work groups, Acad. Management Exec. 17, 96 (2003).

[54] B. R. Johnson and C. J. D'Lauro, After brainstorming, groups select an early generated idea as their best idea, Small Group Res. 49, 177 (2018).

[55] K. McMahon, A. Ruggeri, J. E. Kämmer, and K. V. Katsikopoulos, Beyond idea generation: the power of groups in developing ideas, Creativity Res. J. 28, 247 (2016).

[56] A. Young, Teaching Writing Across the Curriculum,, 4th ed., Landmark Publications in Writing Studies (Pearson Prentice Hall, Upper Saddle River, NJ, 2006).

[57] H Tinberg, Metacognition is not cognition, in Naming What We Know: Threshold Concepts of Writing Studies, edited by L. Adler-Kassner and E. Wardle (University Press Colorado, Boulder, CO, 2015).

[58] N. González, L. C. Moll, and C. Amanti, Funds of Knowledge: Theorizing Practices in Households, Communities, and Classrooms (Routledge, London, 2006).

[59] D. Tannen, Turn-taking and intercultural discourse and communication, in The Handbook of Intercultural Discourse and Communication, edited by C. B. Paulston, S. F. Kiesling, and E. S. Rangel (Blackwell Publishing Ltd., Oxford, 2012), pp. 135-157.

[60] M. E. Brenner, Interviewing in educational research, in Handbook of Complementary Methods in Education Research, edited by J. L. Green, G. Camilli, and P. B. Elmore (Routledge, 2006).

[61] See Supplemental Material at http://link.aps.org/ supplemental/10.1103/PhysRevPhysEducRes.17.010120 for details.

[62] C. Geisler and J. Swarts, Coding Streams of Language: Techniques for the Systematic Coding of Text, Talk, and Other Verbal Data (WAC Clearinghouse, 2019).

[63] K Charmaz, Constructing Grounded Theory: A Practical Guide through Qualitative Analysis (Sage, Thousand Oaks, CA, 2006).

[64] M. Hass and J. Osborn, An emic view of student writing and the writing process, Across the Disciplines 4 (2007), https://wac.colostate.edu/docs/atd/articles/ hass_osborn2007.pdf. 
[65] C. Bazerman, Speech acts, genres, and activity systems: How texts organize activity and people, in What Writing Does and How It Does It: An introduction to Analyzing Texts and Textual Practices, edited by C. Bazerman and P. Prior (Lawrence Erlbaum Associates, Hillsdale, NJ, 2004).

[66] D. Melzer, Assignments Across the Curriculum: A National Study of College Writing (Utah State University Press, 2014).

[67] B. T. William, Literacy practices and perceptions of agency: Composing identities (Taylor \& Francis, London, 2017).

[68] K. Taczak, Reflection is critical for writers' development, in Naming What We Know: Threshold Concepts of Writing
Studies, edited by L. Adler-Kassner and E. Wardle (University Press Colorado, Boulder, CO, 2015).

[69] P. B. Kohl and N. D. Finkelstein, Patterns of multiple representations use by experts and novices during physics problem solving, Phys. Rev. Phys. Educ. Res. 4, 010111 (2008).

[70] R. L. Sternberg, The assessment of creativity: An investmentbased approach, Creativity Res. J. 24, 3 (2012).

[71] L. Bao and K. Koenig, Physics education research for 21st century learning, Discip. Interdiscip. Sci. Educ. Res. 1 (2019). 